\title{
Nutritional Status in Preeclamptic Women: a Case-Control Study in South East of Iran
}

\author{
Mahdieh Sheikhi ${ }^{*}$, Elham Rezaei ${ }^{2}$, Seyede hosniye Hosseini ${ }^{3}$, Mahnaz Shahrakipoor ${ }^{4}$, Monire Sheikhi ${ }^{3}$, Sepideh Soltani $^{5}$ \\ 1- Ph.D Student, Health Deputy, Zahedan University of Medical Sciences, Zahedan, Iran. \\ 2- Food Security Research Center, Department of Clinical Nutrition, School of Nutrition and Food Sciences, Isfahan University of Medical Sciences, \\ Isfahan, Iran. \\ 3- Nutritionist and diet therapy, Zahedan University of Medical Sciences, Zahedan, Iran. \\ 4- Assistant Prof, Department of Biological Statistics, Faculty of Health, Zahedan University of Medical Sciences , Zahedan, Iran . \\ 5- Ph.D Student, Department of Nutrition, School of Public Health, Iran University of Medical Sciences, Tehran, Iran.
}

\section{A B S T R A C T}

Background and Objectives: Beyond the influence of anthropometric, demographic and race, nutrition is considered as one of the most important risk factors for development of preeclampsia (PE). The issue has grown controversial in light of recent reports. This paper, purposes to compare the nutritional and demographic status between PE women and normal pregnancy in south east of Iran.

Materials and Methods: Sixty three normal pregnant and $62 \mathrm{PE}$ women were recruited to be referred to obstetrics and gynecology department of Imam-Ali Hospital, Zahedan, Iran. PE was diagnosed by using international guidelines by the expert gynecologist. Dietary intake, assessed by food frequency questionnaire (FFQ), and anthropometric measurement and medical history were collected by trained gynecologist and dietitians during the second trimester. The logistic regression model was used to assess the correlation between dietary intake and PE risk.

Results: Pregnant PE women compared with normal pregnant women had higher pre-pregnancy body mass index (BMI), cesarean delivery and low birth weight babies. There was an association between grain intake and PE risk $(\mathrm{OR}=2.00 ; 95 \%$ $\mathrm{CI}=1.11-3.61$ ). No association was found between other dietary intakes and PE risk.

Conclusions: Our findings support the association between grain intake and the risk of PE. Further prospective studies are needed to illustrate the link between dietary intake and PE status.

Keywords: Preeclampsia, Nutrition, Diet, Pregnant women

\section{Introduction}

Preeclampsia (PE) is one of the most multisystem disorders in pregnancy, which can lead to maternal and fetal morbidity and mortality $(1,2)$. PE prevalence was reported $2-8 \%$ in the world, mostly in nulliparous women $(1,3)$. The population attributable risk (PAR) for PE found 19.8-35.7\% in all maternal BMI ranges in developing countries and $20.9 \%$ In Iran (2). It is also related to an increased risk of perinatal mortality and is the cause of approximately $10 \%$ of stillbirths and $15 \%$ of preterm births (4). Social-environmental factors, such as maternal age, pre-pregnancy weight and history of previous pregnancy are reported to affect the PE incidence and gestational outcomes (2,5-7). Despite extensive studies and recent evidence to identify the pathophysiology of PE, the etiology of this disorder is questionable and requires further investigation (8). Disturbed placentation, endothelial dysfunction, oxidative stress and a low-grade systemic inflammatory condition are related to the diagnostic criteria for PE, leading to the clinical signs including hypertension, proteinuria and edema after the twentieth week of pregnancy $(9,10)$.

According to recent literature, higher than normal BMI, multiple pregnancies and pregnancy pause $>10$ y, PE family history, autoimmune diseases, young age 
and history of PE in a prior pregnancy as well as insulin resistance, diabetes, dyslipidemia and chronic hypertension are some of the risk factors associated with PE $(4,8,9,11)$. Meta-analysis studies found that obesity and high BMI can predict the risk of PE and small for gestational age newborns $(2,5)$. Lifestyle and dietary modification may reduce the PE risk (2,12-15). Several studies showed that additional energy intake, processed foods, refined grains and sugar-sweetened beverage consumption increases the PE risk $(16,17)$, vice versa more fruits and vegetable consumption, milk and nuts can reduce the risk of PE $(18,19)$. Recommended foods may protect against PE due to high content of fiber, vitamin E, C, D, selenium and zinc, while processed foods and high-fat diet increases free radicals, which can lead to increased inflammation and oxidative stress risk factors $(4,5,20,21)$. There are different evidence regarding micronutrient effects on PE. Several studies reported the positive effects of antioxidant vitamins, while others did not observe a significant difference $(13,3)$. A study was conducted in Iran, demonstrating that higher intakes of energy, as well as lower intakes of vitamin $\mathrm{C}$, magnesium and potassium are positively associated with the risk of gestational hypertension (22).

The relationship between PE and diet have been conducted in different regions, but in the South East of Iran, less attention has been paid to this issue. PE and eclampsia are the second leading cause of maternal and perinatal morbidity and mortality in Iran and is the highest maternal mortality rate in Zahedan (23). The aim of this study was to evaluate nutritional status among pregnant women with and without PE, and possible adverse pregnancy outcomes between pregnant women living in Zahedan.

\section{Materials and Methods}

Subject's selection: We carried out a population based case-control study at the Obstetrics and Gynecology department of the Imam-Ali hospital, from June 2015 to September 2015 in Zahedan. The hospital is a referral focalize for high risk pregnancies and women from diverse social classes were admitted.

A sample size of 68 pregnant women aged 18-35 (20 weeks pregnant) were included for each group based on a power of $80 \%$ at the $5 \%$ significance level. For each PE woman, a normal pregnant woman was randomly selected and were matched together for gestational age, without having multiple gestation and medical problems including chronic hypertension, cardiovascular or renal diseases, diabetes and use of medications. PE was diagnosed by a systolic/diastolic blood pressure of $\geq 140 / \geq 90 \mathrm{mmHg}$ on two occasions more than 4 hours apart after 20 weeks and proteinuria $\geq 300 \mathrm{mg}$ protein in 24 -h urine collection (24).

Pregnant women were excluded if they did not give approval, have a history of pre-existing health conditions including type 1 or type 2 diabetes, younger than 18 years, gestational diabetes, hypertension disorders, heart disease, renal disease or other conditions that require special diets and medications.

Based on the exclusion criteria, 62 participants in $\mathrm{PE}$ and 63 participants in normal pregnancy reached the inclusion criteria. Those with $>70$ blank items on the food frequency questionnaire (FFQ) $(n=6 \mathrm{PE}, \mathrm{n}=5$ normal pregnancy) were excluded. A trained gynecologist and dietitians carried out the survey under standard protocols. The participants were interviewed about demographic information. The participants were followed until delivery time for collecting information on the type of delivery and infant birth weight and other measurements.

Dietary assessment: Dietary intake was assessed using a validated 168 -items semi-quantitative FFQ (25). The FFQ was in a Willet format adjusted for Iranian food items $(26,27)$.This questionnaire was used to ascertain the frequency of food intakes during pregnancy. The FFQ asked about the usual dietary intake in the last six months. Information regarding the intake frequency and portion size was changed into the amount of each food item in grams consumed on average per day. Dietary information was extracted by Nutritionist-4 software (First Databank Inc., Hearst Corp, San Bruno, CA) analyzed by a trained nutritionist. Daily consumption was changed from their unit listed in the FFQ to grams per day.

Statistical analysis: Data analysis was implemented using Statistical Package for the Social Sciences (SPSS) (version 18:0, SPSS Inc., Chicago, IL) software. Non-parametric correlation statistics were used because data failed to meet the assumptions of normality and equal variances. A $p$-value of $<0.05$ was considered as significant. The data were expressed as mean $\pm S D$ or percentages. Statistical significances between percentages were assessed by using the chi-square test. The logistic regression 
model has assessed the correlation between dietary intakes and PE risk.

\section{Results}

Demographic and lifestyle variables are determined in Table 1. The mean age $(\mathrm{P}=0.002)$ and pre-pregnancy $\mathrm{BMI}(\mathrm{P}=0.003)$ was higher in $\mathrm{PE}$ women than in the normal pregnancy group but both were in normal ranges. The women with history of abortion were most likely in the PE compared with normal pregnancy $(23.5 \%$ vs. $17.6 \%, \mathrm{p}=0.36)$.For other variables, no significant differences were observed between PE and normal pregnancy.

According to the outcomes accessed from the FFQ, the mean energy, protein, Carbohydrates and fat intake in normal pregnancy and PE groups were not significant (Table 2) and the average intake of food groups were in the recommended range.

PE women had significantly lower intakes of meat or beans and vegetables $(\mathrm{P}<0.001)$, and higher intakes of grains compared to the normal pregnancy group. None of the women used any supplements other than folic acid and iron that is routinely consumed during pregnancy.

The associations between dietary intakes and odds of PE are presented in table3. After adding energy intake in model 1, the odds of PE became significant in the grain intake. The odds of PE significantly was higher in the grain intake. The other food groups (meat, beans, nuts and egg, milk, vegetables and fruit) were not related to the risk of PE. No significant association was found between the risk of PE and micro or macronutrient intake.

The percentage of cesarean section was significantly higher in women with PE compared to normal pregnant women. (54.4 vs 20.6, p-value $<0.0001)$. Newborn birth weight was significantly lower in the PE group compared to normal pregnancy group $(2572.9 \pm 791.4$ vs $3001.6 \pm 480.1$, pvalue $<0.003$ ), however the mean of newborn weight was more than 2500gr in both groups. None of the women in the normal pregnancy group developed PE after delivery.

Table 1.Characteristics of women with preeclampsia and normal pregnancy

\begin{tabular}{|c|c|c|c|c|}
\hline \multicolumn{2}{|c|}{ Characteristics } & $\begin{array}{l}\text { Preeclampsia } \\
\quad(\mathrm{N}=62)\end{array}$ & $\begin{array}{c}\text { Normal gestation } \\
(N=63)\end{array}$ & p-value \\
\hline \multicolumn{2}{|l|}{ Age (yrs) } & $29.3 \pm 6.7$ & $25.8 \pm 6.2$ & 0.002 \\
\hline \multicolumn{2}{|c|}{ Education (yrs) } & $5.8 \pm 4.9$ & $6.8 \pm 5.2$ & 0.32 \\
\hline \multicolumn{2}{|c|}{ Gestational age (wk) } & $33.1 \pm 2.7$ & $34.6 \pm 2.1$ & 0.63 \\
\hline \multicolumn{2}{|c|}{ Pre-pregnancy BMI $\left(\mathrm{kg} / \mathrm{m}^{2}\right)$} & $22.9 \pm 2.7$ & $21.3 \pm 3.2$ & 0.003 \\
\hline \multicolumn{2}{|c|}{ Primiparous (\%) } & 23.5 & 33.8 & 0.25 \\
\hline \multicolumn{2}{|c|}{ History of abortion (\%) } & 23.5 & 17.6 & 0.36 \\
\hline \multicolumn{2}{|c|}{ History of stillbirth (\%) } & 10.3 & 16.2 & 0.46 \\
\hline \multicolumn{2}{|c|}{ History of twin birth (\%) } & 7.4 & 8.8 & 0.77 \\
\hline \multicolumn{2}{|c|}{ History of preeclampsia (\%) } & 8.8 & 2.9 & 0.14 \\
\hline \multirow[t]{3}{*}{ Ethnic } & Baloch(\%) & 79.4 & 69.1 & \multirow[t]{3}{*}{0.20} \\
\hline & Sistani $(\%)$ & 16.2 & 26.5 & \\
\hline & Other $(\%)$ & 4.4 & 4.4 & \\
\hline \multirow[t]{2}{*}{ Residence } & $\operatorname{Urban}(\%)$ & 76.5 & 75 & \multirow[t]{2}{*}{0.84} \\
\hline & $\operatorname{Rural}(\%)$ & 23.5 & 25 & \\
\hline
\end{tabular}

Table2. Comparison of energy, macronutrient and food intakes in preeclampsia and normal pregnancy

\begin{tabular}{lccc}
\hline Daily intake & $\begin{array}{c}\text { Preeclampsia } \\
(\mathrm{N}=62)\end{array}$ & $\begin{array}{c}\text { Normal pregnancy } \\
(\mathrm{N}=63)\end{array}$ & $P$-value \\
\hline Energy(Kcal) & $2707.8 \pm 1219.9$ & $2777.4 \pm 635.1$ & 0.16 \\
Protein(gr) & $95.1 \pm 46.8$ & $95.4 \pm 28.1$ & 0.35 \\
Carbohydrates(gr) & $409.9 \pm 244.6$ & $403.7 \pm 103.7$ & 0.35 \\
Fat(gr) & $85.6 \pm 31.7$ & $95 \pm 40.1$ & 0.24 \\
Meat, beans, nuts and egg(ounce) & $3.4 \pm 2.4$ & $4.5 \pm 3.2$ & 0.02 \\
Milk (cup) & $2.3 \pm 1.4$ & $2.2 \pm 1.2$ & 0.86 \\
Grains (ounce) & $18.4 \pm 6.5$ & $15.8 \pm 14.3$ & 0.008 \\
vegetables (cup) & $2.5 \pm 1.5$ & $3.5 \pm 1.9$ & 0.001 \\
Fruit (cup) & $2.7 \pm 2.3$ & $3.4 \pm 2.5$ & 0.09 \\
\hline
\end{tabular}


Table 3. Associations between risk of preeclampsia and dietary intakes

\begin{tabular}{|c|c|c|c|}
\hline \multirow[b]{2}{*}{ Daily intake } & \multicolumn{3}{|c|}{ Preeclampsia vs. normal } \\
\hline & $\begin{array}{c}\text { Crude } \\
\text { OR }(95 \% \mathrm{CI})\end{array}$ & $\begin{array}{c}\text { Model 1* } \\
\text { OR }(95 \% \mathrm{CI})\end{array}$ & $\begin{array}{l}\text { Model2** } \\
\text { OR(95\%CI) }\end{array}$ \\
\hline Protein(gr) & $0.98(0.83-1.16)$ & $1.00(0.80-1.26)$ & $1.05(0.82-1.35)$ \\
\hline Carbohydrates(gr) & $0.95(0.91-0.99) \dagger$ & $0.96(0.83-1.11)$ & $0.97(0.84-1.13)$ \\
\hline $\begin{array}{l}\text { Fat(gr) } \\
\text { Meat, beans, nuts and egg(ounce) }\end{array}$ & $\begin{array}{l}0.98(0.94-1.01) \\
0.92(0.39-2.15)\end{array}$ & $\begin{array}{l}1.01(0.75-1.36) \\
0.91(0.38-2.14)\end{array}$ & $\begin{array}{l}1.03(0.76-1.41) \\
0.77(0.31-1.93)\end{array}$ \\
\hline Milk (cup) & $1.31(0.23-7.40)$ & $1.41(0.22-9.06)$ & $1.33(0.20-8.59)$ \\
\hline Grains (ounce) & $2.02(1.13-3.62) \dagger$ & $2.02(1.12-3.63) \dagger$ & $2.00(1.11-3.61) \dagger$ \\
\hline vegetables (cup) & $1.08(0.68-1.71)$ & $1.07(0.67-1.71)$ & $1.03(0.64-1.65)$ \\
\hline Fruit (cup) & $1.83(0.95-3.52)$ & $1.79(0.90-3.55)$ & $1.76(0.88-3.53)$ \\
\hline Vitamin $\mathrm{A}(\mu \mathrm{g})$ & $1.00(0.99-1.00)$ & $1.00(0.99-1.00)$ & $1.00(0.99-1.00)$ \\
\hline Vitamin C (mg) & $0.99(0.98-1.00)$ & $0.99(0.98-1.00)$ & $0.99(0.98-1.00)$ \\
\hline Vitamin $D(\mu g)$ & $0.99(0.95-1.03)$ & $0.99(0.95-1.03)$ & $0.99(0.95-1.02)$ \\
\hline Vitamin E (mg) & $1.05(0.94-1.17)$ & $1.05(0.94-1.17)$ & $1.06(0.94-1.19)$ \\
\hline Thiamin (mg) & $2.40(0.34-16.48)$ & $2.11(0.22-20.33)$ & $1.32(0.12-14.60)$ \\
\hline Riboflavin (mg) & $1.58(0.06-36.10)$ & $1.47(0.05-36.86)$ & $0.97(0.03-27.85)$ \\
\hline Niacin (mg) & $1.08(0.88-1.34)$ & $1.09(0.88-1.35)$ & $1.06(0.86-1.32)$ \\
\hline Vitamin B6 (mg) & $1.02(0.12-8.49)$ & $1.04(0.12-8.73)$ & $1.35(0.15-11.96)$ \\
\hline Folate $(\mu \mathrm{g})$ & $0.99(0.99-1.00)$ & $0.99(0.99-1.00)$ & $0.99(0.99-1.00)$ \\
\hline Zinc (mg) & $0.84(0.54-1.30)$ & $0.85(0.53-1.36)$ & $0.88(0.54-1.42)$ \\
\hline Iron (mg) & $0.94(0.80-1.09)$ & $0.94(0.80-1.09)$ & $0.94(0.80-1.10)$ \\
\hline Copper (mg) & $5.93(0.76-45.90)$ & $5.81(0.74-45.50)$ & $5.24(0.63-42.97)$ \\
\hline Calcium (mg) & $0.99(0.99-1.00)$ & $0.99(0.99-1.01)$ & $0.99(0.99-1.00)$ \\
\hline Magnesium (mg) & $1.00(0.98-1.01)$ & $1.00(0.98-1.01)$ & $1.00(0.98-1.01)$ \\
\hline Potassium(mg) & $1.00(0.99-1.00)$ & $1.00(0.99-1.00)$ & $1.00(0.99-1.00)$ \\
\hline
\end{tabular}

*Adjusted for energy. **Adjusted for energy, pre-pregnancy BMI and age. $\dagger \mathrm{P}$-value $<0.05$ was considered as significant.

\section{Disc ussion}

The association between nutritional status in PE women and normal pregnancy women was investigated in the present study. To our knowledge, this is the first study reporting the relationship between nutritional status and PE in pregnant women living in Zahedan. PE women had significantly lower intakes of meat or beans and vegetables and higher intake of grains compared to the normal pregnancy group but after adjusting for confounder variables, the women in the grain intake were significantly associated with the PE risk. Some studies showed that additional energy intake, processed foods, refined grains and sugar-sweetened beverage consumption increases the PE risk (16,17). A study (28) showed PE women had significantly lower intakes of milk, meat or beans and higher consumption of grains and fruit, vegetables compared to the recommended minimum number for pregnant women. However, in the case control study in Isfahan, PE women had significantly lower intakes of grains and vegetables compared to the normal pregnancy (29). Another study reported that women who had high intakes of vegetables had lower PE risk (30).

A dietary pattern analysis study advised that adherence to a dietary pattern including high intake of fruits, vegetables, vegetable oils, rice and poultry was associated with reduced PE risk, while adherence to a dietary pattern including a high intake of processed foods (such as white bread ,sausages, hamburgers, sugar-sweetened drinks, salty snacks and sweets) increased the PE risk (15).

The other food groups (meat, beans, nuts and egg, milk, vegetables and fruit) were not related to PE risk. None of micro/macro nutrients were linked to risk of PE. In this study none of micro/macro nutrients were 
linked to PE risk. Whereas some studies showed nutrients may be involved in several important steps in the current proposed concepts of the pathogenesis of PE (31). The underlying mechanisms are complex and remain unclear (8-10). To date there has been controversial results in this issue. Several investigations indicated that vitamin and antioxidants decrease with PE incidence and low birth weight (19, 32). Rumbold et al (33) showed a very low intake of vitamin $\mathrm{E}$ in mid to late pregnancy was associated with an increased PE risk and gestational hypertension, the results were adjusted for confounding factors such as maternal age, parity, body mass index (BMI), PE in a previous pregnancy, preterm delivery in a previous pregnancy, family history of PE, smoking status. Limberaki et alfound that high consumption of antioxidant rich foods, increases the total antioxidant status and developed better health (34). A possible explanation for this might be concluded as dietary intake of vitamin C was higher than recommended allowance and fruitvegetable consumption was more than recommended minimum number during pregnancy. Conflicting evidence was shown in the literature as to approve the relationship of intake of vitamins $\mathrm{C}$ and $\mathrm{E}$ with $\mathrm{PE}$. Zhang et al demonstrated that women who consumed $<85 \mathrm{mg}$ of vitamin $\mathrm{C}$ daily (lower than the recommended dietary allowance), as compared with others, experienced doubled the risk of PE (35). However, Klemmensen et al concluded that incidence of PE did not correlate with dietary vitamin $\mathrm{C}$ and $\mathrm{E}$ intake (36). There was a decreasing trend in the incidence of severe PE and eclampsia with increasing dietary vitamin $\mathrm{C}$ intake. However several studies showed any significant relationship $(1,37,38)$.The recent systematic review and meta-analysis by Schoenaker and colleagues reports an association between gestational hypertensive conditions, including PE, and dietary factors, based on observational studies (18). However, randomize trials and subsequent meta-analyses have demonstrated no beneficial effect of antioxidant vitamin supplementation in reducing the risk of developing PE $(37,39)$. WHO recommendation for PE identified important knowledge gaps in this topic (1).

In this regard, pregnancy outcome indicated the relationship of higher maternal age and pre-pregnancy BMI with increasing PE incidence, cesarean section and infants low birth weight. It must be kept in mind that all of the associations were independent of potential confounders' impact.

Recall bias and selection bias existed as the limitations of case-control studies. So we used random selection and detailed statistical formulas for sample size determination to minimize any bias. Another limitation of this study is that the FFQ is developed for dietary assessment of pervious year, but frequency of items for the last 6 months and intake of some seasonal food items may be not considered. This study has some strengths, including accounting for potential confounders, such as energy, BMI, maternal age and history of PE. Our subjects were carefully interviewed by an expert dietician. Demographic and dietary information were analyzed by questionnaires which have been filled by them. However, more prospective surveys with larger sample size are required to confirm the existing findings.

In conclusion, there are several risk factors for PE. It seems that maternal age and pre-pregnancy BMI are some independent predicting factors for the risk of PE. Throughout findings indicate that healthy dietary pattern in this population is associated with positive birth outcomes and decrease PE incidence. Prospective studies are warranted to confirm the reported findings and further analyze associations.

\section{Financial disclosure}

Authors declared no personal or financial conflicts of interest.

\section{Funding/Support}

This study with the ethics committee code" IR.ZAUMS. REC.1392.5835"was supported by Zahedan University of Medical Sciences. Zahedan, Iran.

\section{References}

1. Organization WH. WHO recommendations for prevention and treatment of pre-eclampsia and eclampsia: summary of recommendations. 2011.

2. Rahman M, Abe S, Kanda M, Narita S, Rahman M, Bilano V, et al. Maternal body mass index and risk of birth and maternal health outcomes in low and middle income countries: a systematic review and meta analysis. Obesity Reviews. 2015;16(9):758-70.

3. Xu H, Shatenstein B, Luo Z-C, Wei S, Fraser W. Role of nutrition in the risk of preeclampsia. Nutrition reviews. 2009;67(11):639-57.

4. Allen R, Rogozinska E, Sivarajasingam P, Khan KS, Thangaratinam S. Effect of diet and lifestyle based 
metabolic risk modifying interventions on preeclampsia: a meta analysis. Acta obstetricia et gynecologica Scandinavica. 2014;93(10):973-85.

5. Poorolajal J, Jenabi E. The association between body mass index and preeclampsia: a meta-analysis. The Journal of Maternal-Fetal \& Neonatal Medicine. 2016;29(22):3670-6.

6. de Jongh B, Mackley A, Jain N, Locke R, Paul D. Effects of advanced maternal age and race/ethnicity on placental weight and placental weight/birthweight ratio in very low birthweight infants. Maternal and child health journal. 2015;19(7):1553-8.

7. Ng S-K, Cameron CM, Hills AP, McClure RJ, Scuffham PA. Socioeconomic disparities in prepregnancy BMI and impact on maternal and neonatal outcomes and postpartum weight retention: the EFHL longitudinal birth cohort study. BMC pregnancy and childbirth. 2014;14(1):314

8. Direkvand-Moghadam A, Khosravi A, Sayehmiri K. Predictive factors for preeclampsia in pregnant women: a unvariate and multivariate logistic regression analysis. Acta Biochim Pol. 2012 Jan 1;59(4):673-7 .

9. Pluta R, Ułamek-Kozioł M, Furmaga-Jabłońska W, Czuczwar SJ. Preeclampsia in the 21st century: Unresolved questions concerning etiology. Nutrition. 2015;31(9):1179-81.

10. Bernardi F, Guolo F, Bortolin T, Petronilho F, Dal Pizzol F. Oxidative stress and inflammatory markers in normal pregnancy and preeclampsia. Journal of Obstetrics and Gynaecology Research. 2008 Dec 1;34(6):948-51.

11. Nucci LB, Schmidt MI, Duncan BB, Fuchs SC, Fleck ET, Britto MM. Nutritional status of pregnant women: prevalence and associated pregnancy outcomes. Revista de saude publica. $2001 \mathrm{Dec} ; 35(6): 502-7$.

12. Dodd J, Thangaratinam S. Researchers' position statement on tackling obesity in pregnancy: the International Weight Management in Pregnancy (IWIP) collaboration pleads for public health intervention. BJOG: An International Journal of Obstetrics \& Gynaecology. 2016 Jan 1;123(2):163-4.

13. Dodd JM, O'Brien C, Grivell RM. Preventing preeclampsia-are dietary factors the key? BMC medicine. 2014 Sep 22;12(1):176.

14. Grieger JA, Grzeskowiak LE, Clifton VL. Preconception dietary patterns in human pregnancies are associated with preterm delivery. The Journal of nutrition. 2014 Jul 1;144(7):1075-80.

15. Englund-Ögge L, Brantsæter AL, Sengpiel V, Haugen M, Birgisdottir BE, Myhre R, Meltzer HM, Jacobsson B. Maternal dietary patterns and preterm delivery: results from large prospective cohort study. Bmj. 2014 Mar 4;348:g1446.

16. Colón-Ramos U, Racette SB, Ganiban J, Nguyen TG, Kocak M, Carroll KN, Völgyi E, Tylavsky FA. Association between dietary patterns during pregnancy and birth size measures in a diverse population in Southern US. Nutrients. 2015 Feb 16;7(2):1318-32.

17. Borgen I, Aamodt G, Harsem N, Haugen M, Meltzer HM, Brantsaeter AL. Maternal sugar consumption and risk of preeclampsia in nulliparous Norwegian women. European journal of clinical nutrition. 2012 Aug $1 ; 66(8): 920-5$.

18. Schoenaker DA, Soedamah-Muthu SS, Mishra GD. The association between dietary factors and gestational hypertension and pre-eclampsia: a systematic review and meta-analysis of observational studies. BMC medicine. 2014 Sep 22;12(1):157.

19. Brantsæter AL, Haugen M, Samuelsen SO, Torjusen H, Trogstad L, Alexander J, Magnus P, Meltzer HM. A dietary pattern characterized by high intake of vegetables, fruits, and vegetable oils is associated with reduced risk of preeclampsia in nulliparous pregnant Norwegian women. The Journal of nutrition. 2009 Jun $1 ; 139(6): 1162-8$.

20. Frederick IO, Williams MA, Dashow E, Kestin M, Zhang C, Leisenring WM. Dietary fiber, potassium, magnesium and calcium in relation to the risk of preeclampsia. The Journal of reproductive medicine. 2005 May;50(5):332-44.

21. Bobic MV, Habek D, Habek JC. Perinatal epidemiological risk factors for preeclampsia. Acta clinica Croatica. 2015 Mar;54(1):9-13. PubMed PMID: 26058236.

22. Kazemian E, Dorosti-Motlagh AR, Sotoudeh G, Eshraghian MR, Ansary S, Omidian M. Nutritional Status of Women with Gestational Hypertension Compared to Normal Pregnant Women. Hypertens Pregnancy. 2013 May;32(2):146-56.

23. Imami Afshar N, Jalilvand P, Delavar B, Radpoian L, Azemikhah A, Motlagh M. National maternal mortality surveillance sys tem. Tehran: Tandis Publication. 2010:13-4.

24. Kirbas A, Ersoy AO, Daglar K, Dikici T, Biberoglu EH, Kirbas O, et al. Prediction of Preeclampsia by First Trimester Combined Test and Simple Complete Blood Count Parameters. Journal of clinical and diagnostic research : JCDR. 2015 Nov;9(11):QC20-3.

25. Esmaillzadeh A, Azadbakht L. Major dietary patterns in relation to general obesity and central adiposity among Iranian women. J Nutr 2008 ;138(2):358-63.

26. Mirmiran P, Azadbakht L, Azizi F. Dietary behaviour of Tehranian adolescents does not accord with their nutritional knowledge. Public Health Nutr 2007;10:897901.

27. Willett W. Nutritional epidemiology. USA: Oxford University Press; 1998.

28. Sheykhi M, Paknahad Z, Hasanzadeh A. Dietary nutrient intake and antioxidant status in preeclamptic women. Adv Biomed Res 2015;4:183. 
29. Sheikhi M, Sharifi-Zahabi E, Paknahad Z. Dietary Antioxidant Capacity and Its Association with Preeclampsia. Clinical nutrition research. 2017 Jan $1 ; 6(1): 47-54$.

30. Longo-Mbenza B, Tshimanga KB, Buassa-bu-Tsumbu B, Kabangu MJ. Diets rich in vegetables and physical activity are associated with a decreased risk of pregnancy induced hypertension among rural women from Kimpese, DR Congo. Niger J Med 2008;17:265-9.

31. Xu H, Shatenstein B, Luo Z-C, Wei S, Fraser W. Role of nutrition in the risk of preeclampsia. Nutrition reviews. 2014;67(11):639-57.

32. Chappell LC, Seed PT, Briley AL, Kelly FJ, Lee R, Hunt BJ, Parmar K, Bewley SJ, Shennan AH, Steer PJ, Poston L. Effect of antioxidants on the occurrence of pre-eclampsia in women at increased risk: a randomised trial. The Lancet. 1999 Sep 4;354(9181):810-6.

33. Rumbold AR, Maats FH, Crowther CA. Dietary intake of vitamin $\mathrm{C}$ and vitamin $\mathrm{E}$ and the development of hypertensive disorders of pregnancy. Eur J Obstet Gynecol Reprod Biol 2005;119:67-71.

34. Limberaki E, Eleftheriou P, Vagdatli E, Kostoglou V, Petrou Ch. Serum antioxidant status among young, middle-aged and elderly people before and after antioxidant rich diet. Hippokratia 2012;16:118-23.

35. Zhang C, Williams MA, King IB, Dashow EE, Sorensen TK, Frederick IO, et al. Vitamin C and the risk of preeclampsia-results from dietary questionnaire and plasma assay. Epidemiology 2002;13:409-16.

36. Klemmensen A, Tabor A, Osterdal ML, Knudsen VK, Halldorsson TI, Mikkelsen TB, et al. Intake of vitamin $\mathrm{C}$ and $\mathrm{E}$ in pregnancy and risk of pre-eclampsia: Prospective study among 57346 women. BJOG 2009;116:964-74.

37. Rumbold A, Duley L, Crowther C, Haslam R. Antioxidants for preventing pre-eclampsia. Cochrane Database Syst Rev. 2005;4.

38. Basaran A, Basaran M, Topatan B. Combined vitamin $\mathrm{C}$ and $\mathrm{E}$ supplementation for the prevention of preeclampsia: a systematic review and meta-analysis. Obstetrical \& gynecological survey. 2010 Oct $1 ; 65(10): 653-67$.

39. Cohen JM, Beddaoui M, Kramer MS, Platt RW, Basso O, Kahn SR. Maternal antioxidant levels in pregnancy and risk of preeclampsia and small for gestational age birth: A systematic review and meta-analysis. PloS one. 2015 Aug 6;10(8). 